\title{
Influence of aspect ratio in convection due to nonuniform heating
}

\author{
H. Herrero ${ }^{1}$ and A. M. Mancho ${ }^{2}$ \\ ${ }^{1}$ Departamento de Matemáticas, Facultad de Ciencias Químicas, Universidad de Castilla-La Mancha 13071 Ciudad Real, Spain \\ ${ }^{2}$ Departamento de Física y Matemática Aplicada, Facultad de Ciencias, Universidad de Navarra, 31080 Pamplona, Navarra, Spain
}

(Received 18 September 1997)

\begin{abstract}
The influence of fluid depth in a convection problem in which heating is nonuniform is studied. We consider a vessel that has at the bottom a temperature distribution which has Gaussian shape in the transversal direction and whose surface is open to the atmosphere. Coupled buoyancy and thermocapillary effects are taken into account. The results confirm a stationary bifurcation and a prelude of an oscillatory one as has been observed recently in convection with quasi-one-dimensional heaters. [S1063-651X(98)09506-3]
\end{abstract}

PACS number(s): 47.11.+j, 47.20.Dr, 47.20.Bp

In convection there are situations in which localized effects are able to generate differences in a large scale. In the general problem of turbulence it is important to know how proceses which involve different scales interact since it is thought that effects in a small scale are responsible for behaviors in a larger scale. In our problem we see how an effect in a small scale (localized heating) influences the behavior of a fluid in a larger scale and we compare this localized effect with the global one. We think that the study of localized effects can help the understanding of turbulence. Along these lines the works of Kazarinoff and Wilkowski [1] study thermocapillary flows in axially symmetric float zones. They discuss localized heating that corresponds to the experimental results in Refs. [2-7], in which the heaters have low "heat capacity" and, therefore, it is conceivable that a coupling between the temperature distributions of the heater and the fluid exists. This coupling has been avoided in Ref. [9] and the situation described here would be more closely related to this experiment. In Ref. [8] the primary stationary bifurcation that appears in convection due to nonuniform heating is studied in a vessel for a fixed aspect ratio between the depth and the width of the cell. In this paper we extend the study to several aspect ratios and we look at the influence of this parameter in the problem. The physical situation that we consider is shown in Fig. 1. There is a horizontal fluid layer of depth $d$ ( $z$ coordinate) in a container of length $L(y$ coordinate) and width $l$ ( $x$ coordinate). As shown in the figure $L \gg l$ and for this reason it can be considered to be infinity. The container has a rigid bottom plate and an upper surface open to the atmosphere. A heater is located in the middle of the bottom plate at $x=l / 2$, along the $y$ direction. The width of this heater is much smaller than the width of the container. The heater is at $T_{0}$, and the temperature of the environment is $T_{1}\left(<T_{0}\right)$. Then $\Delta T=T_{0}-T_{1}$ is the local difference of temperatures in the liquid layer just over the heater. When the heater is switched on a temperature distribution which is Gaussian in $x$ direction appears at the bottom.

The behavior of the system is as follows: a basic convective state appears without threshold if a temperature gradient with horizontal component different from zero is applied. It consists of two big rolls parallel to the heater and filling the convective cell. The basic rolls suffer a stationary bifurcation. The threshold for the bifurcation changes with the depth of the fluid in such a way that while depth increases the temperature gradient required decreases. Experimental measures show us that for increasing depths and high values in the applied temperature gradient traveling waves are possible. We determine the basic state numerically and we perform a linear stability analysis on this solution. We find that the basic rolls suffer a stationary bifurcation and we obtain that theoretically the temperature gradient required depends on the depth in the same way as the experiments show. The threshold also depends on the geometry of the wire heater, on the vessel, and on the environment and fluid properties. By increasing depth it appears a complex eigenvalue branch for the appropriate wave number $k$, but at the reported depths it does not destabilize.

Starting from the general hydrodynamic problem our approximations follow [8].

(1) The Oberbeck-Boussinesq approximation usual in convective problems.

(2) The variation of the surface tension as a function of the temperature is approximated by $\sigma(T)=\sigma_{0}-\gamma\left(T-T_{0}\right)$, where $\sigma_{0}$ is the surface tension at temperature $T_{0}, \gamma$ is the constant rate of change of surface tension with temperature ( $\gamma$ is positive for most current liquids).

(3) The length in the $y$ direction is considered infinite.

(4) The free surface is assumed to be undeformable.

(5) The Prandtl number is considered infinite. This allows some simplifications in the velocity potential and in the equations. A very useful approach is to express the velocity field $u$ in terms of a potential as follows: $u=\nabla \times \nabla \times \phi e_{z}$ $+\boldsymbol{\nabla} \times \xi e_{z}$, where $\boldsymbol{\nabla} \times \boldsymbol{\nabla} \times \phi e_{z}$ is the poloidal part and $\boldsymbol{\nabla} \times \xi e_{z}$ is the toroidal part, but if the fluid has an infinite Prandtl number, the equation for $\xi$ has only the solution $\xi$

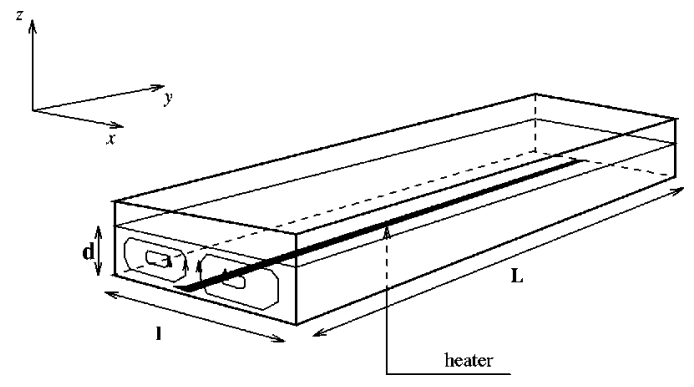

FIG. 1. Problem setup. 


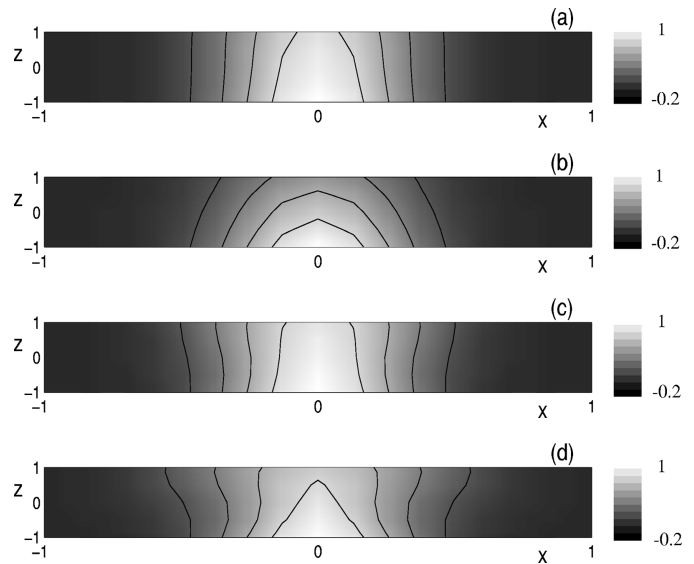

FIG. 2. Isotherms of the basic state before the instability for $\beta$ $=200$ and different $d, h$ numbers and temperature differences $\Delta T_{c}$, the axes are $x$ and $z$. (a) $d=1 \mathrm{~mm}, h=20 \mathrm{~W} /\left(\mathrm{m}^{2}{ }^{\circ} \mathrm{C}\right)$ and $\Delta T$ $=1{ }^{\circ} \mathrm{C} \quad(R=16.614, \quad M=150.654, \quad$ and $B=0.161)$. (b) $d$ $=1 \mathrm{~mm}, h=124 \mathrm{~W} /\left(\mathrm{m}^{2}{ }^{\circ} \mathrm{C}\right)$, and $\Delta T=1{ }^{\circ} \mathrm{C} \quad(R=16.614, \quad M$ $=150.654$, and $B=1.000)$. (c) $d=1 \mathrm{~mm}, h=20 \mathrm{~W} /\left(\mathrm{m}^{2}{ }^{\circ} \mathrm{C}\right)$, and $\Delta T=4.66^{\circ} \mathrm{C} \quad(R=77.420, M=702.056$, and $B=0.161)$. (d) $d$ $=2.5 \mathrm{~mm}, h=20 \mathrm{~W} /\left(\mathrm{m}^{2}{ }^{\circ} \mathrm{C}\right)$, and $\Delta T=1{ }^{\circ} \mathrm{C} \quad(R=259.589, M$ $=376.640$, and $B=0.403)$.

$=0$ and it is sufficient to consider the poloidal part. Therefore the final expression for the velocity field is $u$ $=\left(\partial_{x} \partial_{z} \phi, \partial_{y} \partial_{z} \phi,-\Delta_{1} \phi\right)$, where $\Delta_{1}=\partial_{x}^{2}+\partial_{y}^{2}$. The translational symmetry in the $y$ direction for the basic state implies that all the derivatives in this coordinate are zero. So it is possible in this case to obtain a simpler expression for the velocity field. If we call $\psi=\partial_{x} \phi$, we have $u=\left(\partial_{z} \psi, 0\right.$, $-\partial_{x} \psi$ ).

With these approximations the equations and boundary conditions for the basic state are

$$
\begin{gathered}
\frac{2}{\Gamma} \partial_{z} \psi \partial_{x} \Theta-\frac{2}{\Gamma} \partial_{x} \psi \partial_{z} \Theta=\Delta \Theta, \\
\Delta^{2} \psi-\frac{1}{\Gamma} R \partial_{x} \Theta=0,
\end{gathered}
$$

where $\Delta=\left(1 / \Gamma^{2}\right) \partial_{x}^{2}+4 \partial_{z}^{2}$.

$$
\Theta=\exp \left[-(39 x)^{2} /(\beta)\right] \text { at } z=-1,
$$

$$
\begin{gathered}
\psi=0 \quad \text { at } z=-1, \\
\partial_{z} \psi=0 \quad \text { at } z=-1, \\
B \Theta=-2 \partial_{z} \Theta \quad \text { at } z=1, \\
-4 \partial_{z}^{2} \psi=\frac{M}{\Gamma} \partial_{x} \Theta \quad \text { at } z=1, \\
\partial_{x} \Theta=0 \quad \text { at } \quad x= \pm 1, \\
\psi=0 \quad \text { at }= \pm 1, \\
\partial_{x} \psi=0 \quad \text { at }= \pm 1,
\end{gathered}
$$

where $-1 \leqslant x \leqslant 1,-1 \leqslant z \leqslant 1$. In these equations $\beta$ is the width of the temperature distribution and $\Gamma$ is the aspect ratio which is defined as $\Gamma=l / 2 d$. The boundary conditions at the surface involve the Biot number $B$ and the Marangoni number $M$. The Biot number is $B=h d / K$, where $h$ is the thermal surface conductance and $K$ is the thermal conductivity. It describes the heat interchange at the surface. The Marangoni number is defined as $M=(\gamma \Delta T d) /\left(\kappa \nu \rho_{0}\right)$ where $\gamma$ is the constant rate of change of surface tension with temperature, $\kappa$ is the thermal diffusivity, $\nu$ is the kinematic viscosity, and $\rho_{0}$ is the mean density of the liquid. This number takes account of the thermocapillarity effects.

We have solved numerically these equations with a Chebyshev-collocation method [8]. The fields are approximated by the expansions:

$$
\begin{aligned}
\widetilde{\phi}(x, z) & =\sum_{n=0}^{N-1} \sum_{m=0}^{M-1} a_{n m} T_{n}(x) T_{m}(z), \\
\widetilde{\Theta}(x, z) & =\sum_{n=0}^{N-1} \sum_{m=0}^{M-1} b_{n m} T_{n}(x) T_{m}(z),
\end{aligned}
$$

where $T_{n}, T_{m}$ are the Chebyshev polynomials.

The solution obtained for the basic state can be seen in Fig. 2.

In order to perform a linear stability analysis of the basic state $\left(u_{b}, \Theta_{b}\right)$ we perturb it with a vector field depending on the $x, y$, and $z$ coordinates, in a fully three-dimensional (3D) analysis:

TABLE I. Critical $\Delta T_{c}$ and $k_{c}$ for different orders of expansions for $\beta=200$.

\begin{tabular}{llllclll}
\hline \hline & $h=1240 \mathrm{~W} /\left(\mathrm{m}^{2}{ }^{\circ} \mathrm{C}\right)$ & \multicolumn{2}{c}{$h=124 \mathrm{~W} /\left(\mathrm{m}^{2}{ }^{\circ} \mathrm{C}\right)$} & \multicolumn{2}{c}{$h=20 \mathrm{~W} /\left(\mathrm{m}^{2}{ }^{\circ} \mathrm{C}\right)$} & \\
$d$ & $N \times M$ & $\Delta T_{c}$ & $k_{c}$ & $\Delta T_{c}$ & $k_{c}$ & $\Delta T_{c}$ & $k_{c}$ \\
\hline 1.5 & $23 \times 7$ & 2.51 & 2.85 & 1.01 & 2.40 & 2.45 & 2.25 \\
& $25 \times 7$ & 2.51 & 2.90 & 1.01 & 2.40 & 2.47 & 2.25 \\
& $27 \times 7$ & 2.51 & 2.85 & 1.01 & 2.45 & 2.49 & 2.25 \\
\hline 2.0 & $23 \times 7$ & 2.11 & 2.85 & 0.75 & 2.50 & 1.66 & 2.40 \\
& $25 \times 7$ & 2.11 & 2.90 & 0.75 & 2.45 & 1.68 & 2.35 \\
& $27 \times 7$ & 2.11 & 2.90 & 0.75 & 2.45 & 1.70 & 2.40 \\
\hline 2.5 & $23 \times 7$ & 1.72 & 2.85 & 0.61 & 2.50 & 1.34 & 2.25 \\
& $25 \times 7$ & 1.71 & 2.90 & 0.60 & 2.60 & 1.37 & 2.50 \\
& $27 \times 7$ & 1.71 & 3.00 & 0.60 & 2.60 & 1.39 & 2.50 \\
\hline \hline
\end{tabular}




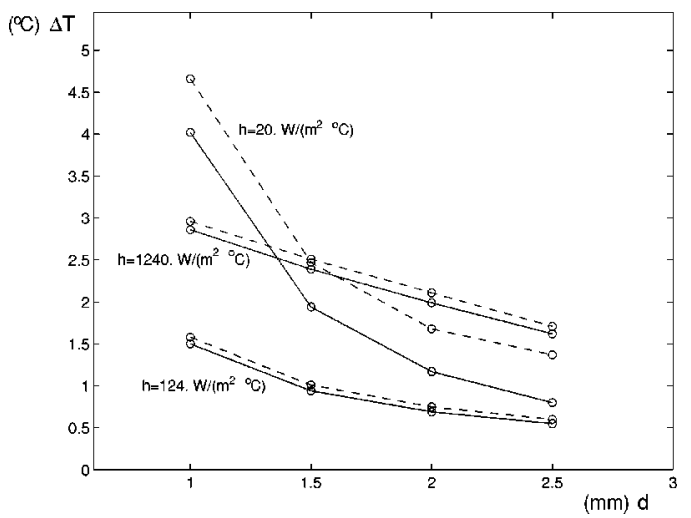

FIG. 3. Critical temperature thresholds $\Delta T_{c}$ as a function of depth for different values of $h\left[h=20,124\right.$, and $\left.1240 \mathrm{~W} /\left(\mathrm{m}^{2}{ }^{\circ} \mathrm{C}\right)\right]$ and $\beta$. The solid line is for $\beta=\infty$ and the dashed line is for $\beta$ $=200$. The circles correspond to the points calculated numerically.

$$
\begin{gathered}
u_{b}(x, z)+\tilde{u}(x, z) e^{\lambda t+i k y}, \\
\Theta_{b}(x, z)+\widetilde{\Theta}(x, z) e^{\lambda t+i k y} .
\end{gathered}
$$

The expressions (13) and (14) are replaced in the basic equations (1)-(10). After eliminating the second order terms we obtain the following eigenvalue problem:

$$
\begin{gathered}
\left(\lambda-\Delta+u_{x} \frac{\partial_{x}}{\Gamma}+2 u_{z} \partial_{z}\right) \widetilde{\Theta}+\left(\frac{2}{\Gamma} \partial_{x} \Theta \partial_{x} \partial_{z}-\partial_{z} \Theta \Delta_{1}\right) \widetilde{\phi}=0 \\
-\Delta^{2} \Delta_{1} \widetilde{\phi}+R \Delta_{1} \widetilde{\Theta}=0 \\
-4 \partial_{z}^{2} \widetilde{\phi}-M \widetilde{\Theta}=0 \quad \text { at } z=1 \\
\widetilde{\phi}=0 \quad \text { at } z=-1,1 \\
\partial_{z} \widetilde{\phi}=0 \quad \text { at } z=-1 \\
2 \partial_{z} \widetilde{\Theta}-B \widetilde{\Theta}=0 \\
\widetilde{\Theta}=0 \quad \text { at } z=-1 \\
\widetilde{\phi}=0 \quad \text { at } x=-1,1 \\
\partial_{x} \widetilde{\Theta}=0 \quad \text { at } x=-1,1 \\
\partial_{x} \widetilde{\phi}=0 \quad \text { at } x=-1,1 \\
\partial_{x}^{2} \widetilde{\phi}=0 \quad \text { at } x=-1,1
\end{gathered}
$$

where the Laplacians are $\Delta=\left(1 / \Gamma^{2}\right) \partial_{x}^{2}+4 \partial_{z}^{2}-k^{2}$ and $\Delta_{1}$ $=\left(1 / \Gamma^{2}\right) \partial_{x}^{2}-k^{2}$. We solve the problem posed numerically with a Chebyshev-collocation method in which convergence has been tested. In order to do this we consider that it is reached if differences of consecutive approximations decrease for increasing order of the expansions and the differences between the $\Delta T_{c}$ calculated with consecutive approximations are less than $0.05^{\circ} \mathrm{C}$. The influence of increasing the order in the $z$ direction expansions $(M)$ is very small,

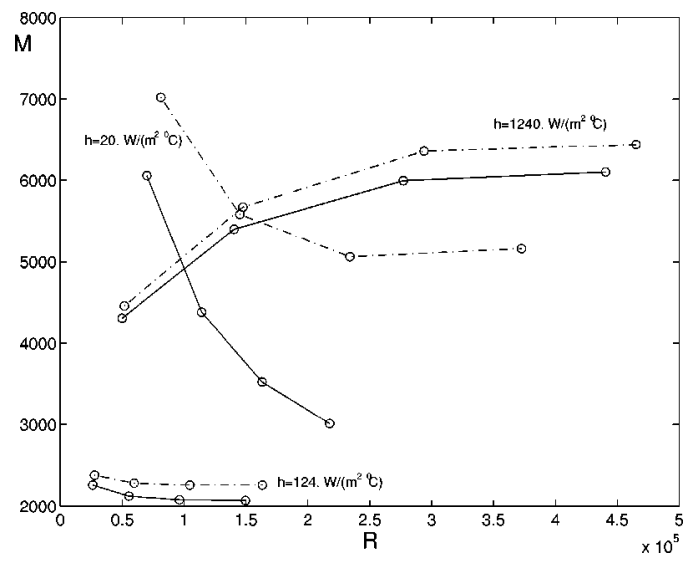

FIG. 4. Critical Marangoni number as a function of Rayleigh number for different values of $h \quad[h=20,124$, and $\left.1240 \mathrm{~W} /\left(\mathrm{m}^{2}{ }^{\circ} \mathrm{C}\right)\right]$ and $\beta$. The solid line is for $\beta=\infty$ and the dashed line is for $\beta=200$. The circles correspond to the points calculated numerically.

and for this reason we only consider the increasing order in the $x$ direction expansions $(N)$. The results can be seen in Table I. From them we see that convergence improves by increasing $h$ or $\beta$ and decreasing $d$. The influence of depth and other parameters, such as the width of the distribution $\beta$ or the thermal surface conductance $h$, in the stability of the basic state is studied by taking different values for them.

In Fig. 3 the solid line shows for $\beta=\infty$ and different values of $h$, the critical thresholds $\Delta T_{c}$ depending on $d$. It can be appreciated that as $d$ increases $\Delta T_{c}$ decreases. The dependence of the critical threshold on the thermal surface conductance is not monotonous; it is minimum for $h$ $=124 \mathrm{~W} /\left(\mathrm{m}^{2}{ }^{\circ} \mathrm{C}\right)$ (i.e., for $\left.d=2, B=2\right)$ and the maximum changes between $h=20 \mathrm{~W} /\left(\mathrm{m}^{2}{ }^{\circ} \mathrm{C}\right)$ (i.e., for $d=2, B$ $=0.3226)$ and $h=1240 \mathrm{~W} /\left(\mathrm{m}^{2}{ }^{\circ} \mathrm{C}\right)$ (i.e., for $\left.d=2, B=20\right)$. We can say the same for $\beta=200$. The results are shown by the dashed line. The difference between both situations is that the critical temperature difference increases while $\beta$ decreases. The same information as in Fig. 3 but with the classical dimensionless numbers $R$ and $M$ as can be seen in Fig. 4.

The value of $d$ affects not only the value of the critical temperature but also the value of the critical wave number.

TABLE II. Critical wave numbers $k_{c}$ depending on $d$ for the two values of the width of the distribution $\beta$ and different values of the thermal surface conductance $h$.

\begin{tabular}{lccc}
\hline \hline$h\left[\mathrm{~W} /\left(\mathrm{m}^{2}{ }^{\circ} \mathrm{C}\right)\right]$ & $d$ & $k_{c}(\beta=200)$ & $k_{c}(\beta=\infty)$ \\
\hline \multirow{3}{*}{1240} & 1.5 & 2.90 & 2.80 \\
& 2.0 & 2.90 & 2.80 \\
& 2.5 & 2.90 & 2.75 \\
\hline \multirow{3}{*}{124} & 1.5 & 2.40 & 2.40 \\
& 2.0 & 2.45 & 2.40 \\
& 2.5 & 2.60 & 2.45 \\
20 & 1.5 & 2.25 & 2.05 \\
& 2.0 & 2.35 & 2.10 \\
& 2.5 & 2.50 & 2.15 \\
\hline \hline
\end{tabular}



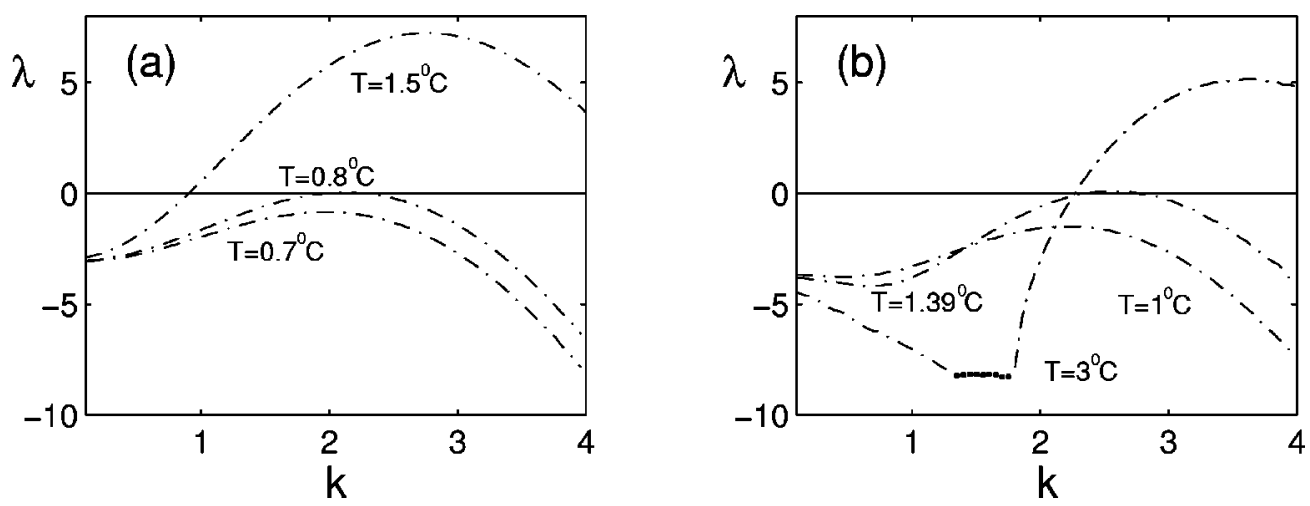

FIG. 5. Eigenvalue curves as a function of the wave number for different temperatures $\Delta T$ and $d=2.5 \mathrm{~mm}$. (a) The heating is uniform $(\beta=\infty)$; (b) the heating is nonuniform $(\beta=200)$. In this case a complex branch appears that is plotted with a thick dotted line.

The main influence in $k_{c}$ is due to $h, k_{c}$ increases when $h$ increases, but for some fixed values of the thermal surface conductance, the values of $k_{c}$ increase for large depths. The lack of homogeneity of the heating also affects the critical wave number since for $\beta=200, k_{c}$ has larger values than the ones obtained for $\beta=\infty$ (see Table II).

An important feature of the eigenvalue curve rises for increasing depths. It consists of the appearance of complex eigenvalues for $k$ which is one-half of that of the stationary patterns but that does not destabilize at the reported depths. We find that if $\beta$ is not infinite and depth takes larger values this effect is favored. In Fig. 5, for uniform heating complex eigenvalues do not appear even after the primary bifurcation. However, if $\beta=200$ for depth $d=2.5 \mathrm{~mm}, \quad h$ $=20 \mathrm{~W} /\left(\mathrm{m}^{2}{ }^{\circ} \mathrm{C}\right)$, and $\Delta T=3{ }^{\circ} \mathrm{C}$ a complex branch appears in the maximum eigenvalue curve. Although a primary bifurcation to traveling waves has not been proven it could be possible that the complex branch of the eigenvalue curve makes them appear as secondary ones.

It is not possible to make a direct quantitative comparison with experiments because in them several parameters are unknown, i.e., the $h$ and $\beta$ numbers. In Ref. [9] for $d=2$ a transition to longitudinal rolls appears for $\Delta T_{c} \approx 17^{\circ} \mathrm{C}$ and we obtain this transition at $\Delta T_{c}=1.68{ }^{\circ} \mathrm{C}, 0.75^{\circ} \mathrm{C}$, and $2.11^{\circ} \mathrm{C}$ for $\beta=200$ and $h=20,124$, and $1240 \mathrm{~W} /\left(\mathrm{m}^{2}{ }^{\circ} \mathrm{C}\right)$, respectively. As $\Delta T_{c}$ increases when $h$ decreases, probably a lower value of $h$ would fit better with the experimental results. However, some qualitative features are recovered. In the experiments described in Ref. [9] the decreasing dependence of the temperature thresholds while the depth increases is qualitatively the same as ours. On the other hand, in the experimental results traveling waves are possible for a wave number half the stationary one. This happens for high depths. Although we do not prove such a bifurcation we find a complex branch in the eigenvalue curve for the appropriate wave numbers.

Our results indicate that the stationary patterns are not interface motions which are excluded from the beginning, but bulk motions. The mechanisms of instability are buoyancy and thermocapillarity, but the patterns are not produced only on the surface, but influence the whole layer of fluid.

We would like to thank M. Bestehorn and I. Mercader for their help in the numerical analysis, and J. Burguete, C. Pérez-García, A. Garcimartín, D. Maza, and H. Mancini for useful comments and discussions. H. H. thanks the Department of Applied Mathematics of the University Complutense of Madrid, where a part of this work was done. This work was partially supported by a Research Project PIUNA (University of Navarra) and DGICYT (Spanish Government) Grant Nos. PB95-0578 and PB96-0534 and by the University of Castilla-La Mancha.
[1] N.D. Kazarinoff and J.S. Wilkowski, Phys. Fluids A 2, 1797 (1990); 1, 625 (1989).

[2] D. Schwabe, R. Velten, and A. Scharmann, J. Cryst. Growth 99, 1258 (1990).

[3] W. Kayser and J. Berg, J. Fluid Mech. 57, 739 (1973).

[4] R. Anthore, P. Flambet, P. Gouesbet, M. Rhazi, and M. Weil, Appl. Opt. 21, 2 (1982).

[5] M. E. Weill, M. Rhazi, and G. Gouesbet, J. Phys. (France) 46, 1501 (1985).

[6] J. Vince and M. Dubois, Europhys. Lett. 20, 505 (1992).

[7] E. Ringuet, C. Roze, and G. Gouesbet, Phys. Rev. E 47, 1405
(1993); E. Ringuet, S. Meunier-Guttin-Cluzel, C. Roze, and G. Gouesbet, J. Phys. (France) II 4, 1243 (1994).

[8] A.M. Mancho, H. Herrero, and J. Burguete, Phys. Rev. E 56, 2916 (1997).

[9] J. Burguete, H. Mancini, and C. Pérez-García, Europhys. Lett. 23, 401 (1993); D. Maza, J. Burguete, and H. Mancini, Int. J. Bifurcation Chaos Appl. Sci. Eng. 4, 1353 (1994); J. Burguete, H. Mancini, D. Maza, and C. Pérez-García (unpublished); J. Burguete and H. Mancini (unpublished); J. Burguete, Ph.D. thesis, Universidad de Navarra, 1995. 